\title{
Raman Spectroscopy as a Clinical Tool for Analysis and Diagnosis of Disease
}

\author{
Michael S. Feld
}

Massachusetts Institute of Technology, GR Harrison Spectroscopy Laboratory, 77 Massachusetts Avenue, Bldg 6-014, Cambridge, MA 02139

We are developing Raman spectroscopy as a clinical tool for analysis and diagnosis of disease. Because each molecule possesses a unique pattern of Raman shifts, the molecular composition of the tissue can be determined. Further, unlike fluorescence, reflectance, and absorption spectroscopies, Raman spectroscopy provides narrow spectral bands, with high information content, that can be assigned to specific molecular vibrations. The data provide direct chemical information that is capable of characterizing a sample both qualitatively and quantitatively.

We have developed quantitative analytical methods to determine the biochemical and morphological composition of cells and tissues from their Raman spectra. These models have been tested on ex vivo tissue and are able to characterize and diagnose atherosclerosis and early cancer with accuracy comparable to that of pathology, without the need for tissue removal, fixation and staining, etc. In addition, we are developing Raman spectroscopy for non-invasive "transcutaneous" blood analysis (i.e. without the need for a needle stick). We have demonstrated that concentrations of multiple analytes in human whole blood and serum can be measured accurately.

For biological samples, near infrared (NIR) radiation is typically employed. NIR excitation reduces background fluorescence, minimizes photothermal degradation, and provides deeper sampling depths. The large penetration depth in human tissue provides the opportunity to observe subsurface structures, regions that cannot be probed using conventional white light endoscopy. Further, eliminating thermal and photochemical damage while probing the maximum volume possible is critical for spectroscopic techniques used in medical procedures.

Despite its rich information content, Raman spectroscopy has not experienced as much clinical investigation as fluorescence. The Raman effect is weak. Typically only about one incident photon in 10 million is converted to a Raman-scattered photon. Hence, the Raman component can be easily overwhelmed by elastic scattering. Signal optimization requires close attention to system design. Considerations based on optical throughput/brightness, characterization of the effective Raman source in the (turbid) tissue, and careful optical design must all be brought together. In addition, the lack of availability of effective optical fiber Raman spectral probes has severely limited clinical applications.

In the past year we have developed a new type of optical fiber probe capable of efficiently collecting the weak Raman signal from arterial tissue and suppressing the intense spectral background generated in the fibers themselves. These probes are flexible, of small diameter, and have greater collection efficiency than traditional open-air Raman systems, while greatly reducing the fiber background plaguing previous Raman probe designs. Figure 1 shows a spectrum of a non-calcified atherosclerotic plaque, which clearly shows a spectral contribution from cholesterol, acquired in 1 second with our Raman probe. For comparison, a spectrum of a 
normal coronary taken with our traditional open-air Raman system in 10 seconds is shown in Figure 2. Note the superior signal-to-noise obtained with the Raman probe. Very recently, these probes have been successfully used to diagnose vascular atherosclerotic lesions in 20 patients undergoing vascular surgery.

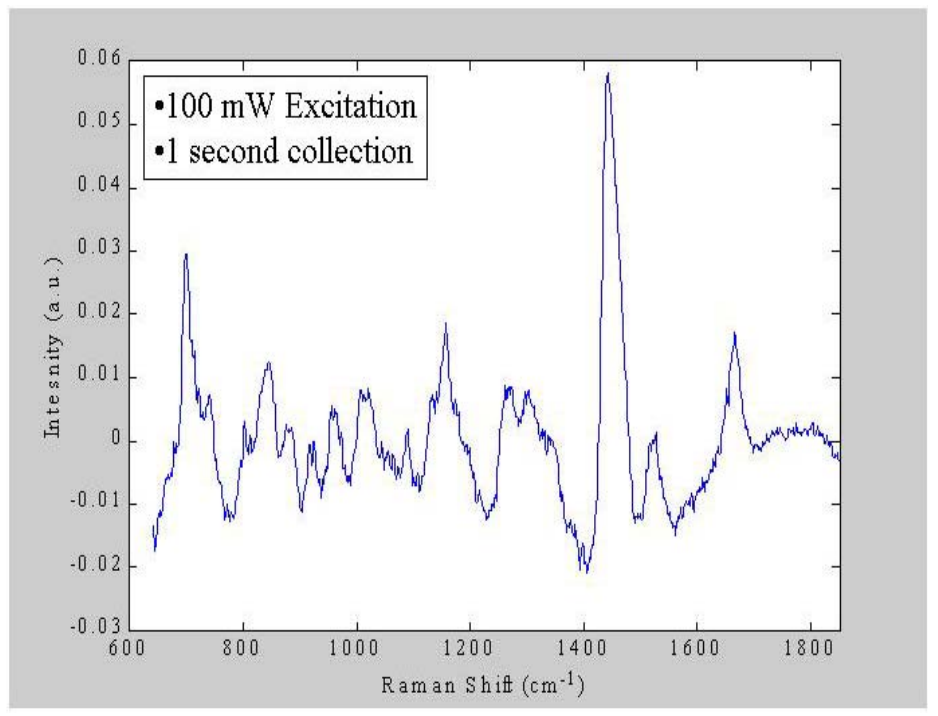

Figure 1. Raman spectrum of a non-calcified atherosclerotic plaque taken with the Raman probe.

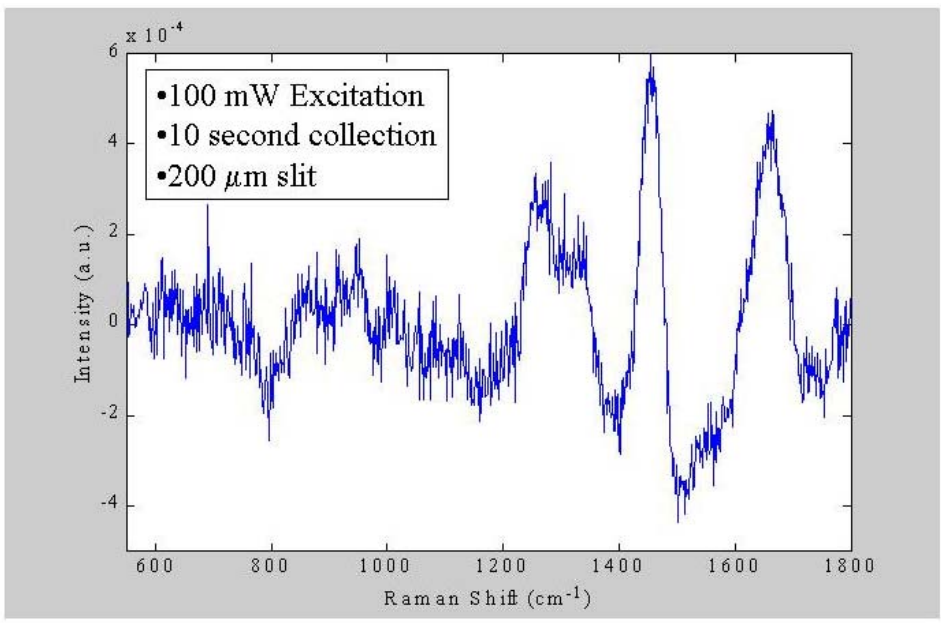

Figure 2. Raman spectrum of a normal coronary artery taken with open-air optics Raman system. 